None of the authors has a financial or proprietary interest in any materials or methods mentioned

Eye (2006) 20, 951-955. doi:10.1038/sj.eye.6702062; published online 26 August 2005

Sir,

\section{Pre-eclampsia resulting in central retinal vein occlusion}

Visual obscurations are common during pregnancy. Photopsias, serous retinal detachments, blurred vision, and cortical blindness has been reported as complications in pre-eclamptic women. Most result in transient visual loss. ${ }^{2}$ However, the combination of blindness and pre-eclampsia remains a rare phenomenon. To our knowledge, we report the first case of central retinal vein occlusion secondary to pre-eclampsia in the puerperium.

\section{Case report}

A 20-year-old woman booked in her first pregnancy at 12 weeks gestation for antenatal care. At booking, her blood pressure was $100 / 55 \mathrm{mmHg}$ and routine bloods were normal. In the past she had a history of a heart murmur. Being symptomatic with shortness of breath and a feeling of extra beats, formal cardiology assessment and transthoracic echocardiogram were requested and found to be normal. The remainder of her antenatal care was uneventful until $39+$ weeks gestation.

At this visit her blood pressure was $130 / 70 \mathrm{mmHg}$ and she had $2++$ of proteinuria on dipstick testing. A mid-stream urine analysis from 1 week previously was clear. She also complained of bilateral leg swelling for the past 2-3 weeks. She was referred to the maternity unit for assessment. On admission her blood pressure profile ranged from $120-170 / 85-100 \mathrm{mmHg}$, with persistent $2++$ proteinuria. She denied any headaches, visual disturbances, or epigastic pain. Examination was unremarkable with normal reflexes and fundoscopy. The baby was well grown and cardiotocography was normal. Routine pre-eclampsia blood tests (FBC, U \& E's, LFT's, uric acid, and clotting) were normal apart from a raised uric acid at $0.40 \mathrm{mmol} / 1$. The patient was admitted and a 24-h urine collection for protein was commenced. The following day her condition remained stable. By the second day her blood pressure was $174 / 88 \mathrm{mmHg}$ and the $24-\mathrm{h}$ urine collection result showed an elevated protein level of $1.52 \mathrm{~g} / 1$ (normal range $<0.3 \mathrm{~g} / 1$ ). Reflexes were hypereflexic with marked ankle clonus. In view of this potential impending eclampsia the decision was made to deliver. The cervix was favourable for surgical induction via forewater amniotomy. Subsequent monitoring of her blood pressure ranged from $135 / 78$ to $186 / 110 \mathrm{mmHg}$. She progressed to an uneventful normal vaginal delivery of a live female infant. During labour and the postnatal period she was kept under close observation, but was not commenced on any antihypertensive nor magnesium sulphate. After 3 days she was discharged with a blood pressure of $130 / 80 \mathrm{mmHg}$, normalised blood tests and reducing oedema.

Approximately 3 weeks postpartum, the patient presented to the Ophthalmology Department with
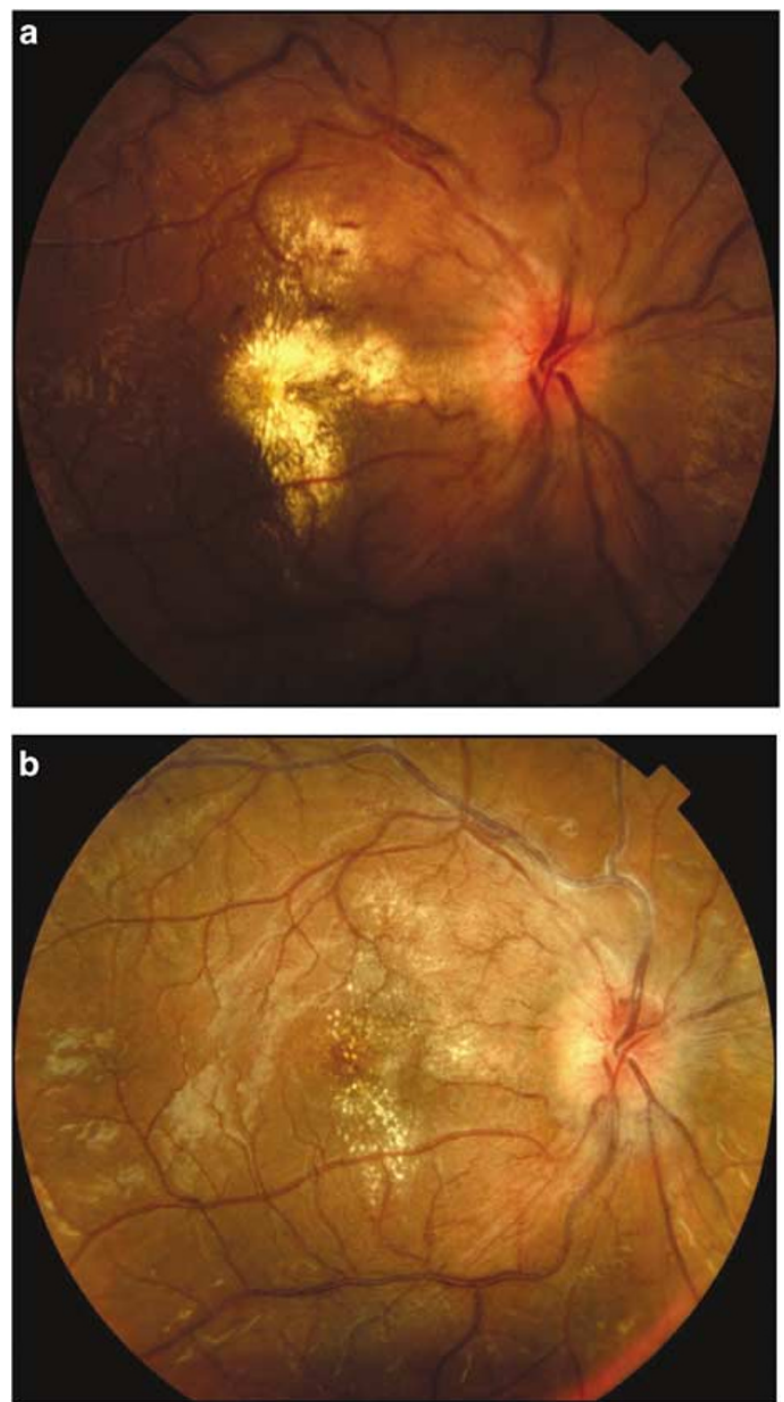

Figure 1 (a) Fundus appearance of the patient at 5 month follow-up. Note the dilated venules, scattered haemorrhages, disc swelling and gross exudation surrounding the macular. (b) Fundus appearance at 9 months showing reduced exudates and disc swelling. 
sudden loss of vision to the right eye. Snellen visual acuity measured $3 / 36$, correcting to $6 / 60$ pinhole in the right eye, and $6 / 5$ unaided in the left. Pupil reactions and anterior segment biomicoscopy were normal, as were the intraocular pressures of both eyes. On dilated

fundoscopy of the right eye, retinal haemorrhages were noted in all four quadrants, with associated gross exudative macular oedema, venous dilation, and mild optic disc swelling (Figure 1). There was no evidence of neovascularisation. Her blood pressure at this time remained within normal limits, at $126 / 74 \mathrm{mmHg}$. A diagnosis of right central retinal vein occlusion was made. Subsequent autoimmune (dsDNA, rheumatoid factor, ANA, antiphospholipid antibodies) investigations, haematological review and thrombophilia screens were normal. At most recent review, 9 months after the loss of vision, the patient's vision has improved to $6 / 9$, with reduced macular exudation and disc swelling.

\section{Comment}

Von Graefe first described retinal changes in preeclamptic women in 1855 . $^{3}$ Pre-eclampsia is generally a disorder of first pregnancies, presenting in upto $5 \% .^{4}$ Transient blindness and visual disturbances have been reported in $25 \%$ of pre-eclamptic women, with only $1-3 \%$ suffering permanent visual loss. ${ }^{5}$

The pre-eclamptic syndrome is primarily a result of placental underperfusion, resulting in generalised endothelial dysfunction. Resultant retinal changes may manifest as decreased retinal to vein ratio, cotton wool spots, haemorrhages, Elschnig spots and serous retinal detachments. The mechanism of such changes remain unknown, but is believed to be a result of a combination of factors, including pre-existing vascular disease, hormonal changes, endothelial damage, alterations in cerebral autoregulation, and hypoperfusion-induced ischaemia. ${ }^{2}$ Jaffe and Schatz ${ }^{6}$ suggested that retinal changes in pre-eclamptic patients maybe partly a result of systemic vascular disease, as vascular changes seem to be paramount in the mechanisms. In their review of 31 patients, Jaffe and Schatz, found a significant relationship between reduced arteriole to vein ratio and pre-eclampsia, suggesting retinal vasospasm and resistance to blood flow as a possible explanation for visual symptoms. The use of magnesium, used in the treatment protocols for severe pre-ecalmpsia, has been shown to dilate retinal arterioles and consequently prevent visual loss from vasospasm. $^{7}$

Loss of cerebral vascular autoregulation may result in orbital vasodilation and hyperperfusion, with resultant increased retinal oedema and serous detachments. ${ }^{8}$
Further, influences of hormonal changes to ocular haemodynamics should not be ignored. Although not yet fully evaluated, oestrogen and other hormones may provide further insight into the pathophysiology of visual loss in pre-eclampsia.

Gonzalvo et $a l^{9}$ reported a case of central retinal vein occlusion associated with pre-eclampsia and HELLP syndrome, a thrombotic microangiopathic vasculopathy, following caesarean section. However, this was attributed to haematological changes associated with HELLP syndrome.

Pathophysiological changes induced by pre-eclampsia may take several weeks to resolve following delivery. Venous thromboembolic disorders must fulfil Virchow's triad of vessel wall abnormality, abnormalities of blood flow and enhanced coaguability of blood. Although, pre-eclamptic vascular changes may account for these changes, permanent visual loss remains a rare entity in this setting, and so, systemic and haematological causes must be excluded. Nevertheless, the chance of visual recovery remains good.

\section{Acknowledgements}

Conflict/Financial interests: None.

\section{References}

1 Sunness JS. The pregnant woman's eye. Surv Ophthalmol 1988; 32: 219-238.

2 Dinn RB, Harris A, Marcus PS. Ocular changes in pregnancy. Obstet Gynecol Surv 2003; 58: 137-144.

3 Duke-Elder S, Dobree JH. Diseases of the retina. In: Duke-Elder S (ed). System of Ophthalmology, Vol, 10. St Louis: CV Mosby, 1967, pp 350-356.

4 Pritchard JA, MacDonald PC, Grant NF. Williams Obstetrics, 17 ed. Norwalk: Appleton-Century-Crofts, 1985, pp 525-560.

5 Dieckmann WJ. The Toxemias of Pregnancy, 2 ed. St Louis: CV Mosby, 1952, pp 240-249.

6 Jaffe G, Schatz H. Ocular manifestations of preeclampsia. Am J Ophthalmol 1987; 103: 309-315.

7 Belfort MA, Saade GR, Moise Jr KJ. The effect of magnesium sulphate on maternal retinal blood flow in preeclampsia: a randomized placebo-controlled study. Am J Obstet Gynaecol 1992; 167: 1548-1553.

8 Ohno Y, Kawai M, Wakahara Y, Kitagawa T, Kakihara M, Arii $Y$ et al. Ophthalmic artery velocimetry in normotensive and preeclamptic women with or without photophobia. Obstet Gynaecol 1999; 94: 361-363.

9 Gonzalvo FJ, Abecia E, Pinilla I, Izaguirre LB, Olivan JM, Honrubia FM et al. Central retinal vein occlusion and HELLP syndrome. Acta Ophthalmol Scand 2000; 78: 596-598.

I Rahman', G Saleemi², D Semple ${ }^{2}$ and P Stanga ${ }^{1}$

${ }^{1}$ Manchester Royal Eye Hospital, Lister Centre, Nelson Street, Manchester, UK 
${ }^{2}$ Department of Obstetrics and Gynaecology, Leighton Hospital, Crewe, UK

Correspondence: I Rahman,

Specialist Registrar,

Manchester Royal Eye Hospital,

Lister Centre, Nelson Street,

Manchester M13 9WL, UK

Tel: +44 161276 5580;

Fax: + 441612726618

E-mail: imran1973@tiscali.co.uk

Eye (2006) 20, 955-957. doi:10.1038/sj.eye.6702065;

published online 5 August 2005

Sir,

Loose cilium in meibomian gland: mimicking early postoperative endophthalmitis

Eyelashes (cilia) are normally arranged in two to three rows on the upper and lower lid, being more numerous and longer in the upper lid. On an average there are 150 lashes on the upper lid and around 75 in the lower lid. ${ }^{1}$ Occasionally lashes may be found in aberrant locations appearing to grow from these unusual locations. The clinical significance of such an occurrence lies in a possible diagnostic difficulty. Some surgeons prefer to have cilia trimmed preoperatively. This may cause loose cilia to get lodged in the eye or adnexa.

We present a case of single cilia lodged in the orifice of meibomian gland, which simulated the symptoms of endophthalmitis after uneventful phacoemulsification.

\section{Case report}

A 53-year-old male underwent uneventful phacoemulsification and foldable posterior chamber intraocular lens implantation in the right eye. On the day preceding surgery, eyelid cilia were trimmed using a pair of scissors and the area was cleaned with antibiotic ointment applied on a sterile cotton-tipped applicator. The patient was advised to wash his face and scalp on the morning of surgery.

At 20 min before surgery the lid margins were cleaned with the cotton-tipped applicator soaked in betadine (5\% $\mathrm{w} / \mathrm{v}$ ) solution. Under aseptic precautions the right eye was cleaned and draped. Sterile disposable eye drape was used, which was in turned towards fornices with the help of a wire speculum, covering the lid margins and the trimmed cilia. Uneventful phacoemulsification was completed using a scleral tunnel incision. No sutures were applied. Antibiotic ointment was applied on the lid margins and the eye patched. The postoperative followup on day one was unremarkable. Acuity of vision was $6 / 9$ compared to preoperative visual acuity of $6 / 36$. On the third postop day the patient complained of redness, pain, watering, and foreign body sensation. Visual acuity had dropped to $6 / 12$. Severity of symptoms raised the suspicion of early postoperative endophthalmitis. On slit-lamp examination the cornea was clear except for a few fluorescein positive superficial punctuate erosions. The anterior chamber was quiet and the intraocular lens was well placed. Fundus examination revealed a media clarity of grade one. On careful examination a cilium was found protruding out of one of the upper meibomian gland orifice (Figure 1a). The tapered end of the hair shaft was hidden within the gland and the trimmed end seemed to rub against the cornea causing the corneal lesions.

The cilium was removed with epilation forceps very smoothly without the need to pluck it out (Figure 1b). No change in the postoperative regimen of topical drops was
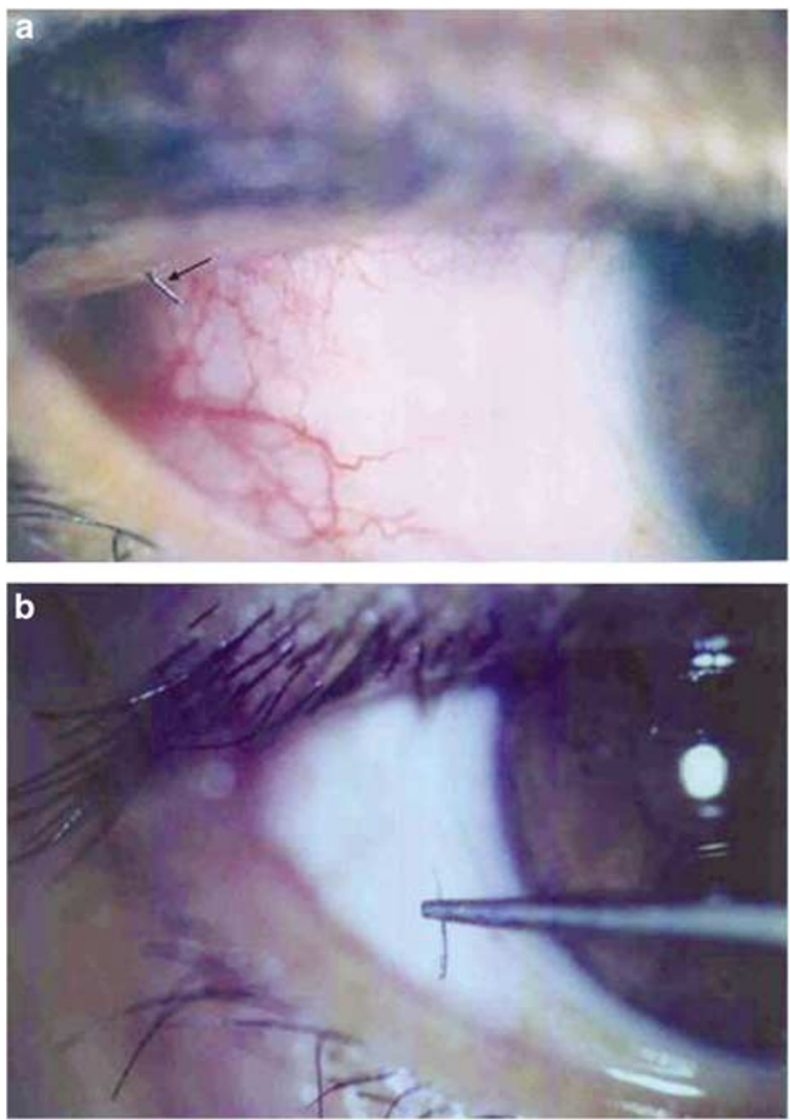

Figure 1 (a) Slit-lamp photo of the right eye showing cilium protruding through the orifice of meibomian gland (arrow). Associated conjunctival congestion is also seen. (b) Eyelash removed with forceps. Note the tapered and the trimmed end of the cilium. 